\title{
Caractérisation et impact du microbiote bactérien respiratoire sur les maladies virales
}

\author{
Maxime Pichon \\ Bruno Lina \\ Laurence Josset \\ Centre hospitalier universitaire de Lyon, \\ Laboratoire de virologie, Institut \\ des agents infectieux (IAI) de Lyon, \\ Centre national de référence des virus \\ respiratoires France Sud, Centre \\ de biologie et de pathologie Nord, \\ Groupement hospitalier Nord \\ F-69317, Lyon cedex 04, France \\ Université Claude Bernard Lyon 1, \\ Faculté de médecine Lyon Est, \\ CIRI, ENS, Inserm U1111 \\ CNRS UMR5308, équipe Virpath, \\ F-69372, Lyon cedex 08, France \\ $<$ maxime.pichon01@chu-lyon.fr>
}

\begin{abstract}
Résumé. Longtemps considéré comme stérile, le tractus respiratoire est maintenant reconnu comme un espace comportant un microbiome à fort potentiel évolutif. L'amélioration des technologies de séquençage permet désormais l'étude de la physiopathologie du microbiome bactérien respiratoire et de ses liens avec la sévérité des infections virales respiratoires. Après une revue des méthodes de caractérisation du microbiote bactérien, en général, et respiratoire, en particulier, cette revue s'attachera à décrire l'impact de ce microbiote sur l'immunité innée et acquise de l'hôte infecté. Nous verrons comment l'exposition virale, survenant dans un contexte d'altération ou de déplétion totale du microbiote respiratoire après antibiothérapie, influence fortement l'évolution clinique de l'hôte infecté. La prise en charge des patients infectés, à risque de complications, pourrait bénéficier de ces nouvelles connaissances. En effet, des études récentes démontrent que de nombreuses voies de réponse de l'hôte à l'infection sont perturbées lorsque le microbiote bactérien est altéré. Son rééquilibrage, par administration de probiotiques ou de molécules innovantes modifiant sa composition, pourrait améliorer le pronostic de l'infection.
\end{abstract}

Mots clés : microbiome respiratoire, infections virales, NGS, ADN16S, métagénomique

\begin{abstract}
Considered for years as sterile, respiratory tract contains very variable bacterial microbiome. Driven by next generation sequencing techniques, microbiome analyses allow a better understanding of viral infection pathophysiology, in terms of incidence and lethality. After a short reminder of microbiome characterization methods, this review will describe its impact on adaptive and innate immunity of the infected host. Studied by viral challenges after antibiotic administration, microbiome depletion has an important impact on clinical outcome of the host. Patient's management and therapeutic choices could exploit results of this field of research, using microbiome characteristics as prognostic biomarkers. Numerous immunologic pathways are impacted by modification of the microbiome and its restoration, using probiotic or innovative molecules could confer better clinical prognostic of viral respiratory diseases.
\end{abstract}

Key words: respiratory microbiome, viral infections, NGS, 16S DNA, metagenomic

(1)

\section{Introduction}

Le microbiote est l'ensemble des micro-organismes présents dans un environnement (ou «niche écologique ») donné, recoupant ainsi la notion, historique, de flore bactérienne. Le microbiome, au sens large, correspond à

Tirés à part : M. Pichon l'ensemble de la niche écologique considérée. Celle-ci inclut à la fois les micro-organismes résidents (bactéries, virus, parasites et champignons) et les facteurs pouvant influencer l'environnement (cellules de l'immunité, protéases cellulaires, etc.). Les méthodes traditionnelles pour détecter le microbiote bactérien reposaient initialement sur des méthodes de culture, faiblement sensibles et fortement chronophages. Au cours des dix dernières années, l'amplification suivie du séquençage de l'ADN codant 
l'ARN16S bactérien a permis d'étudier le microbiote bactérien cutané, des tractus ou des organes, autant quantitativement que qualitativement, et de définir, grâce à la sensibilité de détection ainsi obtenue, comparée aux méthodes de culture, le microbiome [1].

Avec l'émergence de technologies de séquençage à haut débit, ou séquençage dit de "nouvelle génération » (Next Generation Sequencing [NGS]), capables de produire une quantité considérable de données par échantillon, des études de grande ampleur comme celle du Projet microbiome humain (Human Microbiome Project [HMP]) ont permis une avancée importante dans la description des différents microbiomes de l'homme. De nombreuses questions restent néanmoins posées quant à ces microbiomes, notamment sur leur potentielle richesse. Certains auteurs considèrent qu'il y a jusqu'à $10^{14}$ bactéries à la surface ou dans notre organisme, soit dix fois plus que le nombre de nos propres cellules. Ces données restent néanmoins discutées, allant jusqu'à diminuer d'un facteur 10, selon les analyses les plus récentes [2].

À l'instar de l'ensemble des microbiotes décrits chez l'humain, la composition du microbiote du tractus respiratoire est de mieux en mieux définie, ainsi que son rôle physiologique. Notamment, il est fait l'hypothèse qu'il participe aux défenses de l'hôte [3]. Le microbiome des voies aériennes, supérieures ou inférieures, pourrait ainsi agir comme une véritable barrière biologique, participant au maintien de fonctions respiratoires normales et protégeant l'individu de la colonisation ou de l'invasion par des pathogènes extérieurs.

Dans cette revue, nous décrirons les connaissances actuelles sur le microbiome respiratoire dans le cadre des infections respiratoires virales. Nous nous focaliserons sur les données correspondant au microbiome bactérien, du fait du faible nombre d'études réalisées sur le virome et le mycobiome respiratoire (microbiomes viral et fongique, respectivement).

\section{Méthodes d'étude et de caractérisation du microbiome bactérien respiratoire}

Contrairement aux niches écologiques, comme le tractus digestif, depuis longtemps identifiées comme porteuses d'une véritable flore bactérienne, le tractus respiratoire a longtemps été considéré comme stérile, les méthodes de culture dites « classiques » ne permettant pas toujours d'isoler les bactéries anaérobies ou difficiles à cultiver. Depuis l'apparition des technologies de biologie moléculaire, indépendantes d'une étape de culture, le tractus respiratoire inférieur (dont le poumon) est maintenant reconnu comme une niche écologique à part entière, au même titre que la peau ou le tube digestif. Les méthodes d'étude des communautés bactériennes ont considérablement évolué au cours des dernières années, du fait de l'identification d'un gène bactérien universel (codant l'ARN ribosomal 16S) et de l'amélioration progressive des techniques de séquençage, ainsi que l'obtention de puissances de calcul bio-informatique permettant de produire des millions de séquences par échantillon [4].

Le gène codant l'ARN16S est un gène universellement présent chez les procaryotes. Il est d'une longueur d'environ 1,5 kilobases et comporte neuf régions hypervariables (V1 à V9) entourées de dix régions hautement conservées (C1 à C10) (figure 1). Dans un premier temps, il a été analysé et comparé par clonage, par électrophorèse sur gel à gradient de dénaturation (Denaturing Gradient Gel Electrophoresis [DGGE]) ou par l'étude des polymorphismes par restriction de taille (Restriction Fragment Length Polymorphism [RFLP]). Rapidement, ces techniques ont été remplacées par les technologies de séquençage à haut débit $[5,6]$. L'équipe de Carl Woese a utilisé cette stratégie pour classer les bactéries selon leur appartenance à des groupes taxonomiques distincts (Operative Taxonomic Unit [OTU]) révolutionnant ainsi la recherche en écologie microbienne, animale comme environnementale [7]. En effet, la structure du gène codant l'ARN16S autorise la production d'amorces universelles dans des zones conservées, permettant de séquencer certaines parties hypervariables de ce gène (tableau 1 et figure 1). Spécifiques de la plateforme utilisée et d'une longueur de quatre à 12 nucléotides, ces amorces permettent l'attribution (« démultiplexage ») des séquences produites à leur échantillon d'origine, puis le classement en OTU des reads (ou séquences courtes) produits. De nombreux logiciels permettent l'analyse, plus ou moins ouverte, de ces OTU, comme mothur, QIIME ou UPARSE, qui sont les plus utilisés [8-10]. Toutes les étapes du processus de séquençage, comme les différents protocoles d'extraction ou le choix de la polymérase, des amorces et de la région amplifiée, ont un impact sur la qualité des résultats obtenus [11, 12]. De nombreux protocoles et logiciels existent donc pour traiter et vérifier la qualité des séquences produites, sans pour autant qu'une approche consensuelle n'ait encore été admise à ce jour $[9,10,13]$.

Cette description de la population bactérienne, pouvant aller jusqu'au genre ou à l'espèce, via la caractérisation des OTU présents dans un échantillon, ne permet néanmoins pas d'obtenir les informations relatives à l'état physiologique de ces bactéries, élément important pour comprendre le fonctionnement du microbiome. Leung et al., analysant les microbiomes bactériens de l'oropharynx de 22 patients infectés ou non par le virus grippal, ont généré, par métatranscriptomique du génome bactérien (analyse du contenu en ARN), des données complètes sur les gènes de régulation bactériens (gènes impliqués dans la mobilité, la 
Tableau 1 Exemples d'amorces ciblant les régions hypervariables du gène codant l'ARN16S bactérien (adapté de Baker et al. [59]).



Les séquences données à titre indicatif, basées sur l'ADN16S d'E. coli, correspondent aux coordonnées nucléotidiques indiquées entre crochets. Les amorces, sens et antisens, sont toujours indiquées dans le sens 5'-3' et nommées selon leur dénomination usuelle. Les nucléotides en rouge correspondent aux nucléotides constants des régions variables (les verts correspondant aux nucléotides hypervariables). 


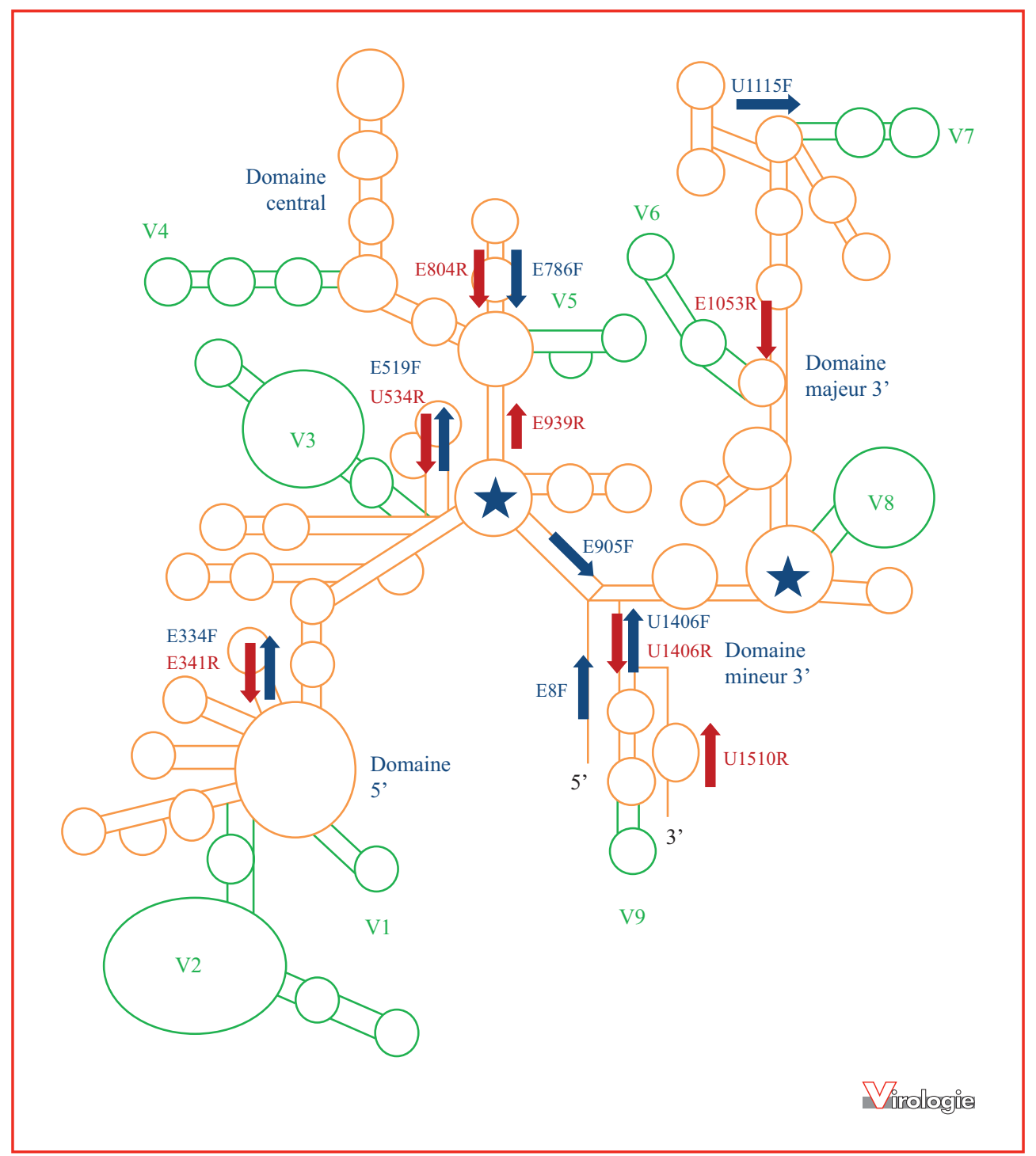

Figure 1. Représentation schématique de la structure secondaire de l'ARN16S bactérien d'E. coli. Vx : régions variables (en vert) permettant la classification des espèces bactériennes présentes dans le microbiome ; Cx : régions constantes (en orange), incluant les amorces de séquençage. Les domaines constants indiqués par l'étoile permettent la distinction entre le domaine 5', le domaine central et les domaines mineurs et majeurs 3'. Les flèches représentent quelques amorces sens (en bleu) et antisens (en orange) utilisables pour les protocoles de séquençage. Les flèches représentent les régions nucléotidiques (en prenant $E$. coli comme génome de référence) correspondant aux amorces (indiquées dans le tableau 1) (bleu : amorces sens et rouge : amorces antisens).

transduction du signal ainsi que le transport ionique et le métabolisme). Il a ainsi été prouvé que les bactéries présentes pouvaient différer non seulement par leur présence ou abondance mais par une activation différentielle de gènes de voies de régulation. Cette différence importante entre bactéries d'un même microbiome pourrait donc permettre d'expliquer leur éventuelle pathogénicité respective [14].

En résumé, si les techniques de NGS permettent d'analyser finement le microbiome des voies aériennes, il est important de garder à l'esprit que l'analyse des séquences de
l'ARN16S ne permet que la détection des génomes (OTU). À moins de combiner ce séquençage génomique à celui des ARNm bactériens, le séquençage des OTU ne permet pas de distinguer les micro-organismes viables ou non [15]. De plus, certaines études pan-microbiennes montrent que la diversité obtenue par séquençage de l'ARN16S est sousestimée comparativement aux approches non ciblées [16]. Il est donc important de distinguer la métagénomique dite «shotgun » (permettant de séquencer l'ensemble des gènes présents dans un échantillon), la métagénomique ciblée (se 


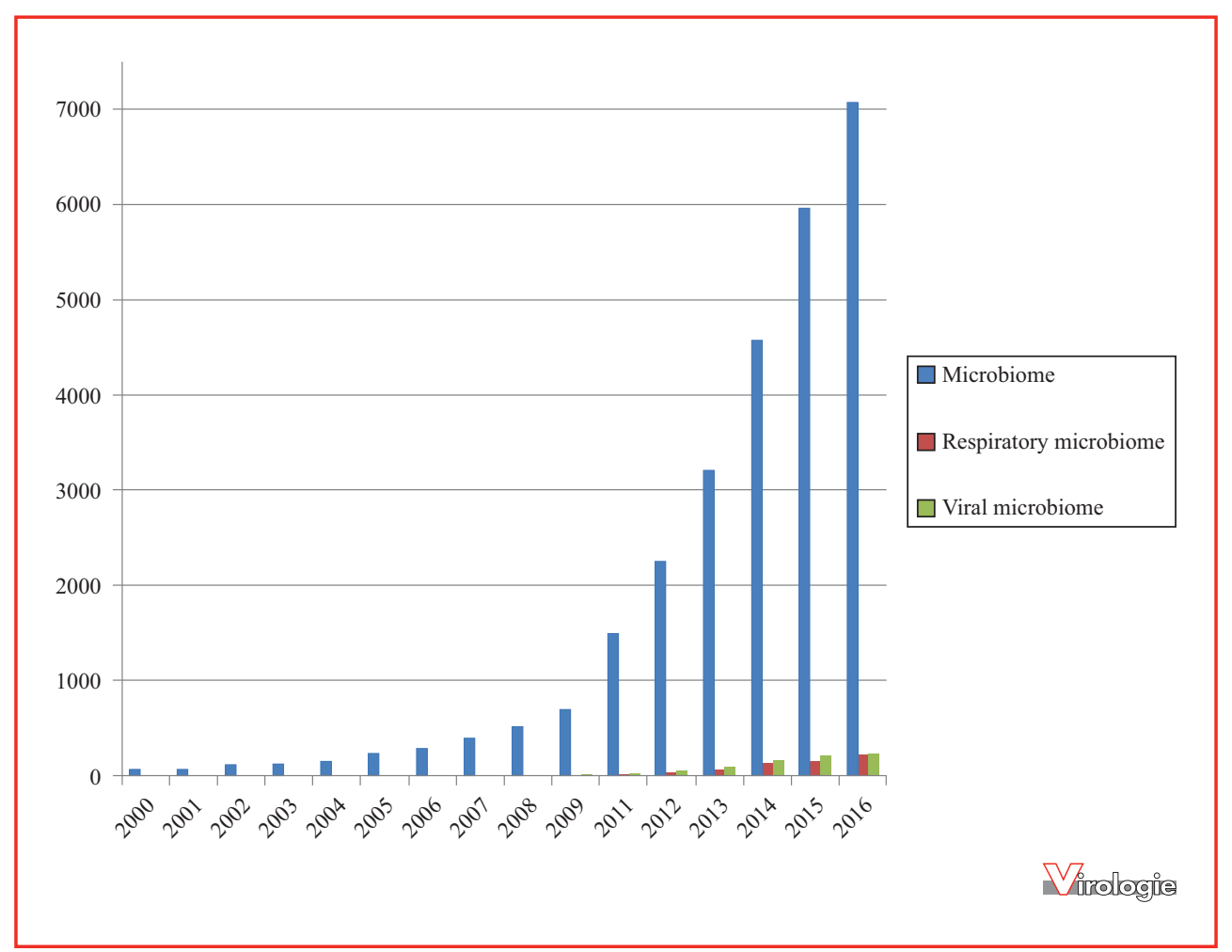

Figure 2. Évolution des données bibliométriques portant sur le microbiome respiratoire de 2000 à 2016 . L'échelle verticale représente le total cumulé d'études correspondant aux mots-clés recherchés et l'échelle horizontale, l'année de publication. Les données en bleu représentent le nombre de publications correspondant au mot-clé « microbiome », les données en rouge représentent le nombre de publications correspondant au mot-clé « respiratory microbiome » et les données en vert correspondent au nombre de publications correspondant au mot-clé « viral microbiome». Le nombre de publications présentent une augmentation notable entre les années 2003 et 2016, correspondant à l'émergence de nouvelles technologies de séquençage. Il est intéressant de constater la différence notable de publications, pouvant s'expliquer par la différence d'intérêt de la communauté scientifique, portant sur les microbiomes respiratoires et non respiratoires (digestif principalement). Cette différence ne diminue pas avec l'explosion du nombre de publications sur le sujet.

limitant à l'étude d'un marqueur, le plus souvent l'ARN ribosomal 16S) et la métatranscriptomique (séquençage de l'ensemble des gènes transcrits ou activés présents dans l'échantillon).

\section{Caractéristiques du microbiome respiratoire sain}

Des études de grande ampleur ont permis d'obtenir des informations importantes quant à la structure et la composition du microbiome du sujet sain au niveau de différentes niches écologiques (figure 2 et tableau 2).

Ces études, conduites aussi sur le microbiome respiratoire, ont permis de distinguer deux microbiomes : «central » et « satellite». Le microbiome respiratoire central (dit core microbiome) est constitué des espèces bactériennes présentes de façon constante dans plus de $95 \%$ des sites prélevés et partagées entre individus sains. Celui-ci comporte les gènes microbiens et les voies métaboliques microbiennes permettant une physiologie stable. À noter qu'il diffère de façon constitutive en fonction de l'âge, du sexe, de l'origine sociale et/ou ethnique des patients, mais qu'il reste dépendant de facteurs environnementaux et/ou extrinsèques tels que la prise de traitements (traitement au long cours ou substitutif) ou l'alimentation [17, 18]. Le mode d'accouchement est un facteur fondamental de variation (quantitative et qualitative) de ce microbiome respiratoire à la naissance [19]. Les enfants nés par césarienne présentent plus longtemps des profils de microbiomes respiratoires centraux dominés par Staphylococcus aureus à la différence des enfants nés par voie basse dont le microbiome respiratoire central évolue plus rapidement vers un profil plus complexe comprenant les genres Moraxella, Corynebacterium ou Dolosigranulum. Ce profil mature permet une meilleure protection contre les infections respiratoires virales [20,21]. Il est, en outre, renforcé par la mise en place d'une alimentation au lait maternel. De même, il apparaît que le microbiome nasopharyngé des enfants prématurés 
Tableau 2 Espèces bactériennes dominant les microbiomes bactériens respiratoires indépendamment du statut pathologique ou non de l'hôte.

\begin{tabular}{|c|c|c|c|c|c|}
\hline \multirow{2}{*}{$\begin{array}{l}\text { Localisation } \\
\text { anatomique }\end{array}$} & \multicolumn{3}{|c|}{ Tractus respiratoire supérieur } & \multicolumn{2}{|c|}{ Tractus respiratoire inférieur } \\
\hline & Cavité buccale & Nasopharynx & Oropharynx & Trachée & Poumon \\
\hline \multirow[t]{2}{*}{$\begin{array}{l}\text { Mode de } \\
\text { prélèvement } \\
\text { privilégié }\end{array}$} & $\begin{array}{l}\text { Écouvillonnage } \\
\text { nez-gorge }\end{array}$ & & & $\begin{array}{l}\text { Aspiration } \\
\text { trachéo- } \\
\text { bronchique }\end{array}$ & $\begin{array}{l}\text { Lavage broncho- } \\
\text { alvéolaire } \\
\text { (biopsie) }\end{array}$ \\
\hline & & \multicolumn{2}{|c|}{ Aspiration nasopharyngée } & & \\
\hline $\begin{array}{l}\text { Bactéries } \\
\text { généralement } \\
\text { retrouvées }\end{array}$ & $\begin{array}{l}\text { Prevotella } \\
\text { Streptococcus }^{\mathrm{a}} \\
\text { Veillonella }^{\mathrm{a}} \\
\text { Haemophilius }\end{array}$ & $\begin{array}{l}\text { Corynebacterium } \\
\text { Streptococcus }^{\mathrm{a}} \\
\text { Veillonella }^{\mathrm{a}} \\
\text { Propionibacterium } \\
\text { Staphylococcus } \\
\text { Moraxella }\end{array}$ & $\begin{array}{l}\text { Prevotella } \\
\text { Streptococcus a } \\
\text { Veillonella } \\
\text { Haemophilius } \\
\text { Fusobacterium }\end{array}$ & $\begin{array}{l}\text { Prevotella } \\
\text { Streptococcus } \\
\text { Veillonella } \\
\text { Cloacibacterium } \\
\text { Helicobacter }\end{array}$ & $\begin{array}{l}\text { Prevotella } \\
\text { Streptococcus }^{\text {a }} \\
\text { Veillonella }^{\mathrm{a}} \\
\text { Pseudomonas } \\
\text { Fusobacterium }\end{array}$ \\
\hline
\end{tabular}

a Espèces présentes de façon constante quelle que soit la région de prélèvement. II est intéressant de constater que les espèces retrouvées sont variables en fonction du site de prélèvement.

est plus hétérogène que celui des enfants nés à terme [22]. Même si la composition du microbiome varie considérablement au cours de la première année de vie, les profils bactériens centraux convergent progressivement, mais très précocement, vers un profil mature, dès l'âge de deux mois, qui reste stable par la suite. Par opposition au microbiome central, le microbiome périphérique, dit « satellite » correspond aux $5 \%$ restants et est sujet à des variations rapides et réversibles au cours des différentes altérations physiopathologiques. Ces altérations sont très diverses comme des maladies aiguës ou chroniques, une grossesse, la sénescence ou le développement de l'enfant. Le microbiome respiratoire périphérique (plus que le microbiome central) semble donc être le reflet de l'exposition de l'individu aux situations extérieures pouvant modifier son microbiome, comme une prise ponctuelle d'antibiotiques, une infection, mais aussi une modification du mode d'alimentation ou des habitus de vie [19, 21-23]. Ces quelques pourcents de variation du microbiome pour sa portion «satellite »étant soumis à changement rapide, un profil de microbiome périphérique d'un individu est donc difficilement comparable à celui d'un autre, justifiant de réaliser pour cela des études appariées et/ou longitudinales pour en étudier les modifications.

\section{Impact du microbiote respiratoire bactérien dans la pathogenèse des maladies virales respiratoires}

Le tractus respiratoire est en contact constant avec l'environnement extérieur [23]. Historiquement, les études portant sur les micro-organismes détectés dans l'appareil respiratoire ont analysé les prélèvements à la recherche d'agents pathogènes. L'avènement des technologies de séquençage à haut débit a progressivement permis de décrire, puis de caractériser, la flore bactérienne résidente commensale afin d'en étudier l'impact sur les réponses immunitaires acquises ou innées le long de l'arbre respiratoire [24]. En 2016, parmi plus de 7000 articles répondant au mot-clé "microbiome », une faible proportion d'entre eux traitait du microbiome respiratoire, en particulier, ou de l'évolution du microbiome, en général, au cours des infections virales (figure 2). Il n'existe que peu de données dans ce domaine de recherche et elles concernent principalement le microbiome bactérien à l'exception de rares publications (1 à $2 \%$ ) qui ont étudié les composantes virales (virome) et fongiques (mycobiome) du microbiome (figure $3 B$ ).

Après un premier temps de caractérisation de la composition et des variations physiologiques du microbiome respiratoire "sain », les études les plus récentes se sont focalisées sur les modifications rencontrées dans des situations dites «pathologiques », qu'il s'agisse d'une infection ou non (figure 3A). Les infections respiratoires aiguës sont majoritairement causées par des virus. En effet, moins de $30 \%$ des causes retrouvées dans les infections respiratoires basses (atteintes des bronches ou des poumons) de l'adulte ne sont pas virales [25]. Cette revue est donc limitée aux descriptions des altérations entraînées par les infections virales sur le tractus respiratoire.

Leung et al. ont étudié, par séquençage complet des génomes bactériens, le microbiome pulmonaire des patients infectés par le virus grippal, le comparant aux microbiomes de patients non infectés possédant les mêmes caractéristiques démographiques [14]. Cette analyse montre que les patients infectés ont un microbiome pulmonaire 

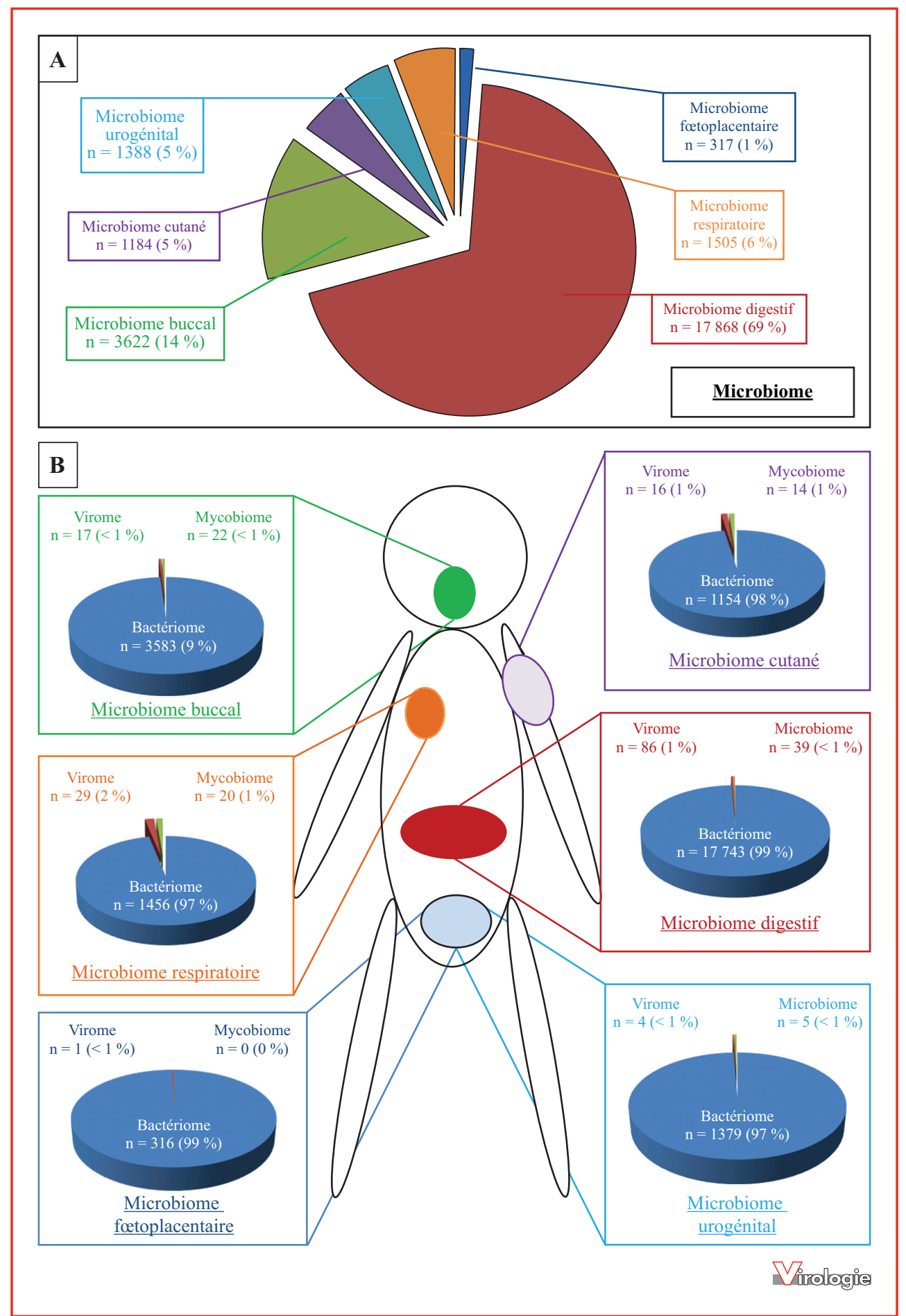

Figure 3. Répartition des publications scientifiques concernant les microbiomes par site anatomique. A) Répartition des publications en fonction de la niche écologique. B) Répartition des publications en fonction des différentes composantes bactériennes, virales et mycotiques du microbiome. À noter que les recherches à propos du microbiome buccal correspondaient aux mots-clés «microbiome» et « oral»; du microbiome respiratoire à « microbiome » et « respiratory» ou « pulmonary» ; du microbiome fœtoplacentaire à « microbiome » et «fetal » ou «placenta »; du microbiome cutané à « microbiome » et «skin»; du microbiome digestif à «microbiome » et « gut » ou « digestive» ; du microbiome urogénital à « microbiome » et « urinary» ou « genital » ou « penis » ou «vaginal ». 
constitué de bactéries exprimant plus de gènes améliorant leurs mobilités (chimiotactisme, flagelles) et leurs capacités de régulation du métabolisme ou de transcription que les bactéries du microbiome des patients «sains». Ces données de métatranscriptomique comparative confortent l'idée de l'existence d'un processus de sélection des microorganismes métaboliquement capables de s'adapter à un écosystème changeant au sein d'un microbiome ouvert. Ces espèces bactériennes, notamment par l'altération du tapis mucociliaire, seraient capables de se déplacer dans les voies aériennes inférieures et de produire des métabolites immunomodulateurs limitant la réponse de l'hôte à l'infection virale. Ces bactéries pourraient donc impacter fortement le devenir clinique, péjoratif ou non, des hôtes infectés.

L'inflammation excessive suivant l'infection virale contribuant au désordre respiratoire observé, un microbiome capable de favoriser une certaine tolérance immunitaire pourrait potentiellement aider à limiter les dégâts tissulaires dus à l'exacerbation de la réponse inflammatoire. Grâce à un modèle murin, Wang et al. ont pu démontrer l'importance d'un microbiome respiratoire intègre sur la tolérance d'un organisme au virus grippal [24]. Pour cela, ils ont utilisé des souris SPF (Specific Pathogen Free) connues pour être plus sensibles aux infections grippales (amaigrissement plus important, taux de survie inférieur...) que les souris sauvages du même génotype élevées dans un environnement naturel non stérile. Dans le modèle de souris SPF, la colonisation du tractus respiratoire supérieur par des bactéries commensales permettait de réduire considérablement les altérations pulmonaires liées à l'infection par le virus influenza. Cette colonisation diminuait la létalité de l'infection grippale via la modification du phénotype de réponse monocytaire, induisant un phénotype d'activation macrophagique moins destructeur [26]. Abt et al. ont également montré que les souris sauvages prétraitées par un cocktail d'antibiotiques, dénaturant ainsi leur microbiome « naturel », présentaient une morbidité accrue lors d'une infection par le virus pandémique A (H1N1) apparu en 2009 (A (H1N1) pdm09), avec une perte de poids et une désaturation en oxygène importantes [27]. La sévérité de ces atteintes était associée à deux facteurs qui étaient :

- une diminution de la capacité de migration des cellules dendritiques, indispensable pour une réponse immunitaire efficace ;

- une diminution de la concentration en cellules lymphocytaires $\mathrm{T}$ spécifiques au niveau tissulaire, contribuant à terme à maintenir une charge virale locale élevée.

Toujours dans un modèle murin, Wu et al. ont montré que l'administration per os d'un traitement probiotique restaurait significativement la réponse immunitaire de souris traitées aux antibiotiques en permettant une meilleure activation des voies TLR-7 et NFкB [26]. Cette voie de signalisation, impliquée dans la reconnaissance des
ARN viraux simple brin, favorise en effet la clairance virale.

L'ensemble de ces données recueillies chez l'animal suggère que la pathogenèse d'une infection par le virus grippal chez un hôte au microbiome altéré est en partie liée à une réponse immunitaire locale moins efficace. Réciproquement, la présence d'un microbiome « intègre » permet de maintenir une réponse innée efficace ainsi qu'une réponse adaptative appropriée contre le virus influenza. La viabilité des micro-organismes composant ce microbiome « bénéfique » ne semble néanmoins pas indispensable. En effet, il a été possible de restaurer expérimentalement, dans un modèle murin, une réponse immunitaire adaptative efficace après administration de lipopolysaccharides (LPS) chez un hôte sain au microbiome " altéré » [29]. L'hypothèse mécanistique est que le microbiome déclenche l'activation de nombreux récepteurs de reconnaissance de motifs moléculaires exprimés par les pathogènes (Pathogen-Associated Molecular Patterns [PAMPS]), stimulant les leucocytes au niveau local comme systémique.

Comme indiqué précédemment, il est maintenant admis que la diversité bactérienne composant le microbiome respiratoire se stabilise à l'approche de la première année de vie. Ce microbiome, par la diversité des micro-organismes qui le constituent, impacte la physiologie de l'hôte ainsi que son immunité [30-34]. La présence de bactéries du genre Moraxella dans ce microbiome semble associée au risque d'une plus grande sévérité de l'infection par le virus respiratoire syncytial (VRS). En retour, le VRS est lui-même capable d'altérer fortement le microbiome respiratoire de l'hôte [21, 35-37]. Durant l'hiver, les coinfections VRS/Moraxella ne sont pas rares, ces deux pathogènes co-circulant durant la période la plus froide de l'année. La bronchiolite à VRS se complique fréquemment d'une otite moyenne d'étiologie bactérienne, suggérant que l'infection virale puisse faire le lit de la surinfection bactérienne. Ce type d'interaction «virus-bactérie » a aussi été démontré de longue date pour les surinfections pneumococciques de la maladie grippale [38]. De nombreux mécanismes d'interaction entre bactéries (principalement le pneumocoque) et virus influenza ont ainsi été mis en évidence depuis leur première description au cours de la pandémie de 1918 (revus dans [39, 40]). Ceux-ci vont de l'interaction directe virus-bactérie, via une activité sialidase du pneumocoque aidant à la libération des virions néoformés, à l'impact anatomique, par destruction de la barrière épithéliale et altération de la structure du surfactant et de l'arbre bronchiolaire, ou immunitaire lié à une dysfonction des réponses innées et adaptatives. De manière générale, il a été montré que les microbiomes nasopharyngés à forte dominance d'Haemophilus influenzae et de Streptococcus, par opposition aux microbiomes riches en $S$. aureus, étaient associés à des réponses immunitaires 
exacerbées de l'hôte lors des infections respiratoires. Cette exacerbation est à l'origine d'une augmentation du taux d'hospitalisation, du fait d'une plus grande sévérité de la maladie au niveau respiratoire, confortant l'hypothèse de l'implication du microbiome dans la réponse inflammatoire aux infections virales [41]. Toutefois, pour l'instant, les études réalisées n'ont fait qu' entrevoir une partie des mécanismes complexes associant le microbiome et la réponse immunitaire.

\section{Impact clinique du microbiome respiratoire}

\section{Utilisation thérapeutique dans le cadre de la prise en charge des infections virales}

L'impact du microbiome dans la pathogenèse des infections respiratoires virales est donc fortement suspecté. Par analogie avec des thérapies du tractus gastro-intestinal, il semble envisageable de moduler le microbiome respiratoire. Nembrini et al. ont, par exemple, développé un modèle murin d'administration directe d'Escherichia coli dans les voies aériennes [42]. Ces inoculations ont montré un effet bénéfique sur l'inflammation locale, fortement impliquée dans la pathogenèse des infections respiratoires, limitant ainsi la symptomatologie clinique. De même, un essai clinique dirigé par Bruzzese et al. a montré l'influence positive de l'administration de Lactobacillus rhamnosus (à distance de la sphère respiratoire car administré oralement) sur le nombre et la sévérité des exacerbations de mucoviscidose [43]. Bien que nécessitant d'être validée par des études de cohorte de taille suffisante, le bénéfice de la modification thérapeutique du microbiome respiratoire dans le cadre de maladies respiratoires chroniques semble fortement suggéré par ces études. Néanmoins, peu d'études se sont intéressées à la modification du microbiome respiratoire dans le cadre des infections virales aiguës.

Certains auteurs ont néanmoins montré que l'augmentation temporaire de l'inflammation des voies aériennes par administration d'antigènes bactériens pourrait être une approche thérapeutique des infections à virus influenza [29]. Dans un modèle murin, il a été aussi montré que des animaux prétraités par des aérosols de lysats bactériens (contenant du LPS) présentaient une réponse inflammatoire plus rapide et plus performante quant à l'obtention de la clairance virale au cours d'une infection virale ultérieure. La réponse induite par le LPS diminuait l'altération tissulaire liée à l'infection virale améliorant la survie des animaux infectés [44]. Ces données restent encore contestées dans le cadre spécifique de l'infection par les virus influenza. En effet, il est admis que l'inflammation générée par l'infection virale est responsable de la majorité des manifestations cliniques observées ; or l'utilisation de traitements immunomodulateurs n'a pas montré d'efficacité dans le cadre d'essais cliniques. La situation semble donc plus complexe. À l'instar de ce qui a déjà été étudié pour la prise en charge des sepsis sévères, la maîtrise de la réponse inflammatoire n'a d'intérêt que lors de la phase précoce de la maladie. Une large part de la difficulté du contrôle de l'inflammation au cours de l'infection grippale est ainsi liée au fait qu'elle varie en fonction au cours du temps, des étapes précoces aux phases tardives de la maladie [45].

De façon similaire, l'activation des TLR-2, 6 et 9, au moyen d'un prétraitement par des agonistes synthétiques, permettait de réduire le titre viral mesuré dans les infections par les virus parainfluenza dans les prélèvements respiratoires, sans pour autant permettre de réduire la symptomatologie d'hyperréactivité bronchique [46]. Enfin, l'administration de toxines inactivées d'E. coli a démontré son efficacité pour constituer une protection contre l'infection par VRS et les virus grippaux, tout comme l'administration de L. rhamnosus avant la mise en contact avec le VRS [47, 48]. Ces lactobacilles permettraient de protéger contre l'excès d'inflammation au cours de l'infection, modifiant à la fois la production de nombreux médiateurs pro-inflammatoires (interférons et interleukines) et l'activation des cellules dendritiques via l'expression de CD103 et CD11b [49].

Plusieurs études cliniques ont tenté de démontrer l'intérêt de l'utilisation des prébiotiques (composés non digestibles favorisant la croissance ou l'activité des bactéries commensales) et/ou des probiotiques (administration directe de bactéries de la flore), modifiant le microbiome respiratoire en présence, pour la prise en charge des infections virales $[28,50-54]$. Dans un essai clinique randomisé en double insu sur une cohorte de nourrissons finlandais, Luoto et al. ont exploré l'effet de l'administration de L. rhamnosus (probiotique) ou d'un mélange de galacto-oligosaccharide polydextrose (prébiotique connu pour favoriser la croissance des lactobacilles et des bifidobactéries) sur la survenue d'infections virales. Ces deux stratégies préventives ont permis de réduire l'incidence des infections respiratoires virales symptomatiques. L'effet observé était très net sur les infections à rhinovirus mais non détectable pour celles à VRS.

Bien que ces premières études soient très encourageantes, le chemin à parcourir est encore long avant de pouvoir utiliser ces approches « d'enrichissement bactérien » du microbiome respiratoire, en vue de prévenir ou de traiter une infection virale respiratoire, et nécessitera encore de nombreuses études impliquant des cohortes de taille importante.

\section{Utilisation en biomarqueurs}

Très rapidement, il a été imaginé d'utiliser le microbiome comme un marqueur de risque pour la survenue d'une 
infection respiratoire sévère. En effet, avant même la description du concept de microbiome, il avait été observé que la détection puis l'augmentation de la proportion de certaines espèces bactériennes (Pseudomonas aeruginosa, par exemple) dans le tractus respiratoire signaient un tournant péjoratif dans l'évolution de la maladie chez les patients atteints de mucoviscidose [55].

La connaissance du microbiome d'un individu pourrait permettre de prédire le niveau de risque de celui-ci vis-à-vis des agressions respiratoires bactériennes ou virales. Même si les données sont encore limitées, il a récemment été décrit que le portage nasal de pneumocoque était statistiquement positivement corrélé à la séropositivité au Métapneumovirus chez l'enfant [56]. Les auteurs concluent ainsi, après vérification dans un modèle cellulaire, à l'implication de cette bactérie dans la susceptibilité d'un organisme, ici un enfant, à l'infection par le Métapneumovirus.

Dans une étude publiée en 2017, notre équipe a étudié les microbiomes des enfants présentant des infections grippales de sévérité variable. Nous avons pu mettre en évidence, chez des enfants infectés par le virus influenza A ou B, que plusieurs OTU (S. aureus, Prevotella, Lachnospiracea incertaesedis, Streptobacillus, Porphyromonas, Granulicatella, Veillonella, Fusobacterium et Haemophilus spp.) étaient présentes, dès le début de la maladie, dans des proportions différentes et que cela avait des conséquences sur le devenir clinique du patient infecté. À l'exception de $S$. aureus, toutes ces OTU étaient retrouvées en quantités plus abondantes chez les patients évoluant vers des formes sévères de grippe, et ce dès le prélèvement initial [57]. La détection de ces OTU conjointement à celle du virus grippal pourrait conduire à proposer une prise en charge adaptée dès le début de l'infection. L'orientation initiale des patients, optimisée du fait de l'existence ou non d'un risque identifié de complications respiratoires, permettrait d'améliorer leur prise en charge. Une étude multicentrique, débutée en 2017, permettra de confirmer la réalité de cette différence et surtout l'utilité d'un tel biomarqueur pour la prise en charge des enfants grippés. Le microbiome pourrait donc servir, à terme, de marqueur pronostique en aidant à la prise en charge précoce de patients à risque.

Bien qu'aucune relation causale entre les caractéristiques du microbiome nasopharyngé et la réponse aux infections virales ne puisse être déduite de ces études, les données actuellement disponibles suggèrent néanmoins que l'étude du microbiome pourrait permettre d'obtenir un facteur prédictif des conséquences cliniques de cette infection. L'utilisation des caractéristiques du microbiome dans le cadre la prise en charge des patients infectés pourrait donc être envisagée. Réciproquement, une modification du microbiome bactérien, par vaccination ou administration, pourrait avoir des conséquences sur les maladies infectieuses, qu'elles relèvent ou non d'une stratégie de prévention vaccinale [58, 59].

\section{Conclusions}

Les études réalisées à ce jour semblent montrer que le microbiome bactérien respiratoire régule plusieurs étapes de la réponse immunitaire à l'infection par un virus respiratoire. Le microbiome respiratoire ayant été étudié jusqu' ici dans des modèles in vitro, des modèles d'infection chronique ou sur des populations standardisées (populations immunodéprimées ou néonatales), il est difficile d'extrapoler les résultats obtenus à la population générale. Il semble toutefois acquis que les micro-organismes constituant le microbiome humain influencent fortement la physiologie humaine et la physiopathologie des infections virales respiratoires.

Les données physiopathologiques disponibles suggèrent néanmoins une possible utilisation des connaissances sur le microbiome dans le domaine de la santé humaine. En effet, ce microbiome étant à la fois cause et reflet d'une détérioration possible de la maladie, son utilisation comme marqueur ou comme outil thérapeutique semble être possible en clinique humaine. La détermination de biomarqueurs de sévérité, par la démocratisation et l'amélioration continue des techniques de séquençage à haut débit, permettra d'améliorer plus finement la prise en charge des patients, en vue d'une «médecine personnalisée ». De même, la correction du microbiome satellite, variable selon les conditions environnementales, pourrait permettre une amélioration thérapeutique ou pronostique des maladies infectieuses, comme c'est le cas pour les maladies inflammatoires.

Les domaines de recherche sur le microbiome constituent un immense champ d'investigation aussi bien à l'échelle du pathogène isolé qu'en tant que communauté microbienne complexe. Ces études permettront surtout de comprendre les interactions avec les autres composantes, aussi bien virales que fongiques, du microbiome. Les informations disponibles sur ces deux dernières composantes restent en effet très limitées, mais augurent potentiellement de nombreuses avancées scientifiques à court terme.

Liens d'intérêts : les auteurs déclarent ne pas avoir de lien d'intérêt en rapport avec cet article.

\section{Références}

1. Iwai S, Fei M, Huang D, et al. Oral and airway microbiota in HIVinfected pneumonia patients. J Clin Microbiol 2012; 50 : 2995-3002.

2. Sender R, Fuchs S, Milo R. Revised Estimates for the Number of Human and Bacteria Cells in the Body. PLOS Biol 2016; 14 : e1002533. 
3. Segal LN, Blaser MJ. A brave new world : the lung microbiota in an era of change. Ann Am Thorac Soc 2014; 11 Suppl 1: S21-7.

4. Di Bella JM, Bao Y, Gloor GB, Burton JP, Reid G. High throughput sequencing methods and analysis for microbiome research. $J$ Microbiol Methods 2013;95:401-14.

5. Muyzer G, Smalla K. Application of denaturing gradient gel electrophoresis (DGGE) and temperature gradient gel electrophoresis (TGGE) in microbial ecology. Antonie Van Leeuwenhoek 1998; 73 : 127-41.

6. Liu WT, Marsh TL, Cheng H, Forney LJ. Characterization of microbial diversity by determining terminal restriction fragment length polymorphisms of genes encoding 16S rRNA. Appl Environ Microbiol $1997 ; 63: 4516-22$.

7. Woese CR, Fox GE. Phylogenetic structure of the prokaryotic domain : the primary kingdoms. Proc Natl Acad Sci U S A 1977; 74 : 5088-90.

8. Hamady M, Walker JJ, Harris JK, Gold NJ, Knight R. Error-correcting barcoded primers for pyrosequencing hundreds of samples in multiplex. Nat Methods $2008 ; 5: 235-7$.

9. Schloss PD, Westcott SL, Ryabin T, et al. Introducing mothur : open-source, platform-independent, community-supported software for describing and comparing microbial communities. Appl Environ Microbiol $2009 ; 75: 7537-41$.

10. Caporaso JG, Kuczynski J, Stombaugh J, et al. QIIME allows analysis of high-throughput community sequencing data. Nat Methods $2010 ; 7: 335-6$.

11. Ahn J-H, Kim B-Y, Song J, Weon H-Y. Effects of PCR cycle number and DNA polymerase type on the $16 \mathrm{~S}$ rRNA gene pyrosequencing analysis of bacterial communities. J Microbiol Seoul Korea 2012; 50 : 1071-4.

12. Albertsen M, Karst SM, Ziegler AS, Kirkegaard RH, Nielsen PH. Back to Basics-The Influence of DNA Extraction and Primer Choice on Phylogenetic Analysis of Activated Sludge Communities. PloS One $2015 ; 10$ : e 0132783 .

13. Bakker MG, Tu ZJ, Bradeen JM, Kinkel LL. Implications of pyrosequencing error correction for biological data interpretation. PloS One $2012 ; 7$ : e44357.

14. Leung RK-K, Zhou J-W, Guan W, et al. Modulation of potential respiratory pathogens by $\mathrm{pH} 1 \mathrm{~N} 1$ viral infection. Raoult D, éditeur. Clin Microbiol Infect 2013; 19:930-5.

15. Sze MA, Hogg JC, Sin DD. Bacterial microbiome of lungs in COPD. Int J Chron Obstruct Pulmon Dis 2014; 9: 229-38.

16. Engel P, Stepanauskas R, Moran NA. Hidden Diversity in Honey Bee Gut Symbionts Detected by Single-Cell Genomics. Richardson PM, éditeur. PLoS Genet 2014; 10 : e1004596.

17. Erb-Downward JR, Thompson DL, Han MK, et al. Analysis of the Lung Microbiome in the « Healthy » Smoker and in COPD. Bereswill S, éditeur. PLOS ONE 2011; 6 : e16384.

18. Huse SM, Ye Y, Zhou Y, Fodor AA. A core human microbiome as viewed through 16S rRNA sequence clusters. PloS One 2012; 7 : e34242. 19. Chu DM, Ma J, Prince AL, et al. Maturation of the infant microbiome community structure and function across multiple body sites and in relation to mode of delivery. Nat Med $2017 ; 23: 314-26$.

20. Biesbroek G, Tsivtsivadze E, Sanders EAM, et al. Early respiratory microbiota composition determines bacterial succession patterns and respiratory health in children. Am J Respir Crit Care Med $2014 ; 190: 1283-92$.

21. Teo SM, Mok D, Pham K, et al. The infant nasopharyngeal microbiome impacts severity of lower respiratory infection and risk of asthma development. Cell Host Microbe 2015 ; 17 : 704-15.

22. Perez GF, Pérez-Losada M, Isaza N, et al. Nasopharyngeal microbiome in premature infants and stability during rhinovirus infection. J Investig Med 2017; 65 : 984-90.

23. Lynch SV. Viruses and microbiome alterations. Ann Am Thorac Soc $2014 ; 11$ Suppl 1 : S57-60.
24. The NIH HMP Working Group, Peterson J, Garges S, Giovanni M, et al. The NIH Human Microbiome Project. Genome Res 2009; 19: 2317-23.

25. Jain $\mathrm{P}$, Jain A, Prakash S, et al. Prevalence and genotypic characterization of human parvovirus B19 in children with hemato-oncological disorders in North India. J Med Virol 2015 ; 87 :303-9.

26. Wang J, Li F, Sun R, et al. Bacterial colonization dampens influenzamediated acute lung injury via induction of M2 alveolar macrophages. Nat Commun 2013; 4 .

27. Abt MC, Osborne LC, Monticelli LA, et al. Commensal Bacteria Calibrate the Activation Threshold of Innate Antiviral Immunity. Immunity 2012;37: 158-70.

28. Wu S, Jiang Z-Y, Sun Y-F, et al. Microbiota Regulates the TLR7 Signaling Pathway Against Respiratory Tract Influenza A Virus Infection. Curr Microbiol 2013; 67:414-22.

29. Ichinohe $T$, Pang IK, Kumamoto $Y$, et al. Microbiota regulates immune defense against respiratory tract influenza A virus infection. PNAS USA $2011 ; 108: 5354-9$.

30. Koenig JE, Spor A, Scalfone N, et al. Succession of microbial consortia in the developing infant gut microbiome. Proc Natl Acad Sci U S A $2011 ; 108$ Suppl $1: 4578-85$.

31. Planer JD, Peng Y, Kau AL, et al. Development of the gut microbiota and mucosal IgA responses in twins and gnotobiotic mice. Nature $2016 ; 534: 263-6$.

32. Bokulich NA, Chung J, Battaglia T, et al. Antibiotics, birth mode, and diet shape microbiome maturation during early life. Sci Transl Med $2016 ; 8: 343 \mathrm{ra} 82$.

33. Yatsunenko T, Rey FE, Manary MJ, et al. Human gut microbiome viewed across age and geography. Nature $2012 ; 486: 222-7$.

34. Desai MS, Seekatz AM, Koropatkin NM, et al. A Dietary FiberDeprived Gut Microbiota Degrades the Colonic Mucus Barrier and Enhances Pathogen Susceptibility. Cell 2016; 167 : 1339-53.e21.

35. Furusawa $\mathrm{Y}$, Obata $\mathrm{Y}$, Fukuda $\mathrm{S}$, et al. Commensal microbe-derived butyrate induces the differentiation of colonic regulatory $\mathrm{T}$ cells. Nature $2013 ; 504: 446-50$.

36. Arpaia N, Campbell C, Fan X, et al. Metabolites produced by commensal bacteria promote peripheral regulatory T-cell generation. Nature $2013 ; 504: 451-5$.

37. Beura LK, Hamilton SE, Bi K, et al. Normalizing the environment recapitulates adult human immune traits in laboratory mice. Nature $2016 ; 532: 512-6$.

38. McCullers JA, Bartmess KC. Role of Neuraminidase in Lethal Synergism between Influenza Virus and Streptococcus pneumoniae. J Infect Dis $2003 ; 187: 1000-9$.

39. McCullers JA. The co-pathogenesis of influenza viruses with bacteria in the lung. Nat Rev Microbiol 2014; $12: 252-62$.

40. Pichon M, Lina B, Josset L. Impact of the Respiratory Microbiome on Host Responses to Respiratory Viral Infection. Vaccines 2017; 5.

41. de Steenhuijsen Piters WAA, Heinonen S, Hasrat R, et al. Nasopharyngeal Microbiota, Host Transcriptome, and Disease Severity in Children with Respiratory Syncytial Virus Infection. Am J Respir Crit Care Med 2016; $194: 1104-15$.

42. Nembrini C, Sichelstiel A, Kisielow J, et al. Bacterial-induced protection against allergic inflammation through a multicomponent immunoregulatory mechanism. Thorax $2011 ; 66: 755-63$.

43. Bruzzese E, Raia V, Spagnuolo MI, et al. Effect of Lactobacillus GG supplementation on pulmonary exacerbations in patients with cystic fibrosis : a pilot study. Clin Nutr 2007; $26: 322-8$.

44. Tuvim MJ, Evans SE, Clement CG, et al. Augmented lung inflammation protects against influenza A pneumonia. PloS One 2009; 4 : e4176.

45. Monneret G, Venet F. Sepsis-induced immune alterations monitoring by flow cytometry as a promising tool for individualized therapy. Cytometry B Clin Cytom 2016;90:376-86. 
46. Drake MG, Evans SE, Dickey BF, Fryer AD, Jacoby DB. Toll-like receptor-2/6 and Toll-like receptor-9 agonists suppress viral replication but not airway hyperreactivity in guinea pigs. Am J Respir Cell Mol Biol $2013 ; 48: 790-6$.

47. Norton EB, Clements JD, Voss TG, Cárdenas-Freytag L. Prophylactic administration of bacterially derived immunomodulators improves the outcome of influenza virus infection in a murine model. $J$ Virol $2010 ; 84: 2983-95$.

48. Williams AE, Edwards L, Humphreys IR, et al. Innate imprinting by the modified heat-labile toxin of Escherichia coli (LTK63) provides generic protection against lung infectious disease. J Immunol $2004 ; 173: 7435-43$

49. Chiba E, Tomosada Y, Vizoso-Pinto MG, et al. Immunobiotic Lactobacillus rhamnosus improves resistance of infant mice against respiratory syncytial virus infection. Int Immunopharmacol 2013;17: 373-82.

50. Arslanoglu S, Moro GE, Boehm G. Early supplementation of prebiotic oligosaccharides protects formula-fed infants against infections during the first 6 months of life. J Nutr 2007; 137 : 2420-4.

51. Luoto R, Ruuskanen O, Waris M, et al. Prebiotic and probiotic supplementation prevents rhinovirus infections in preterm infants : a randomized, placebo-controlled trial. J Allergy Clin Immunol 2014 ; 133 : 405-13.

52. Rautava $S$, Salminen $S$, Isolauri E. Specific probiotics in reducing the risk of acute infections in infancy ? a randomised, double-blind, placebocontrolled study. Br J Nutr 2009; $101: 1722$.
53. Taipale $T$, Pienihekkinen $K$, Isolauri $E$, et al. Bifidobacterium animalis subsp. lactis BB-12 in reducing the risk of infections in infancy. Br J Nutr $2011 ; 105: 409-16$.

54. Maldonado J, Cañabate F, Sempere L, et al. Human milk probiotic Lactobacillus fermentum CECT5716 reduces the incidence of gastrointestinal and upper respiratory tract infections in infants. J Pediatr Gastroenterol Nutr 2012; 54:55-61.

55. Mayer-Hamblett N, Rosenfeld M, Gibson RL, et al. Pseudomonas aeruginosa in vitro phenotypes distinguish cystic fibrosis infection stages and outcomes. Am J Respir Crit Care Med 2014 ; 190 : 289-97.

56. Verkaik NJ, Nguyen DT, de Vogel CP, et al. Streptococcus pneumoniae exposure is associated with human metapneumovirus seroconversion and increased susceptibility to in vitro HMPV infection. Clin Microbiol Infect $2011 ; 17: 1840-4$.

57. Langevin S, Pichon M, Smith E, et al. Early nasopharyngeal microbial signature associated with severe influenza in children : a retrospective pilot study. Journal of General Virology 2017 ; 98, doi: 10.1099/jgv.0.000920.

58. Biesbroek G, Wang X, Keijser BJF, et al. Seven-valent pneumococcal conjugate vaccine and nasopharyngeal microbiota in healthy children. Emerg Infect Dis 2014; 20 : 201-10.

59. Tarabichi Y, Li K, Hu S, et al. The administration of intranasal live attenuated influenza vaccine induces changes in the nasal microbiota and nasal epithelium gene expression profiles. Microbiome 2015; $3: 74$.

60. Baker GC, Smith JJ, Cowan DA. Review and re-analysis of domainspecific 16S primers. J Microbiol Methods 2003 ; 55 : 541-55. 